\title{
MORSE THEORY AND NON-MINIMAL SOLUTIONS TO THE YANG-MILLS EQUATIONS
}

\author{
By \\ Hong-Yu WANG
}

\begin{abstract}
By generalizing a method of Taubes, we use Morse theory to find the higher critical points of Yang-Mills functional.
\end{abstract}

Topology and Analysis name mathematical subjects with robust interaction, often along the basic theme: Study relationship between the critical points of functional and the topology of function space. We consider in this article a vector type non compact variational problem-the Yang-Mills equations, and we raise the question of proving the existence of "true critical points" for this functional, in a framework where the Palais-Smale condition does not hold.

This article should be considered as sequel to [27], where the most of the notations and the terminology were introduced. The reader may find that the exposition in [13], [22], [24], [26], and [27] are useful introductions to Morse theory for the Yang-Mills equations. The main purpose of this paper is that it clearly explains the background and motivation, and gives a method for finding the non-minimal solutions to the Yang-Mills equations on a compact oriented 4-manifold.

In our main result, we suppose that there is a known isolated non-minimal Yang-mills field ("isolated means that the Hessian of Yang-Mills functional is non-degenerate). We then use the min-max method to produce infinitely many other non-minimal Yang-Mills fields.

THEOREM. Let $M$ be a compact oriented Riemannian 4-manifold. Let $A_{0}$ be an isolated non-minimal Yang-Mills connection on $M$ with the structure group $S U(2)$ such that $d\left|P_{ \pm} F_{A_{0}}\right|=0$ along a simple closed geodesic and $\left|P_{ \pm} F_{A_{0}}\right|>0$ on this geodesic. Then there is a constant $K>0$ such that for any positive even integer $k>K$, there exists an irreducible non-minimal Yang-Mills $S U(2)$ connection with the same degree as $A_{0}$.

Received November 1, 1995.

Revised March 29, 1996. 
Theorem is proved by first using a gluing procedure to construct an approximate solution space $N(k)$ that involve many parameters, in our construction $N(k)$ is diffeomorphic to

$$
\prod_{i=1}^{2 k}\left\{(0,1) \times F_{M}^{i} \times S U(2) S U(2)\right\} / \Gamma_{A_{0}} \times \Sigma_{k} \times \Sigma_{k}
$$

where $F_{M}^{i}$ are the frame bundles $\wedge_{ \pm}^{2} M$ of self-dual and anti-self-dual forms on $M, \Gamma_{A_{0}}$ is the isotropy group of connection $A_{0}$, and $\Sigma_{k}$ is the symmetric group on $k$ letters. One then shows that one can produce a new non-minimal solution as a small perturbation of some approximate solution $A$ on $N_{k}$.

It should be remarked that in the case of finding the self-dual solutions Taubes [22] constructed many "higher degree" solutions over the generic 4manifolds. In a certain limit, these solutions have the curvature localized near a finite set of points, with approximately one unit of "topological charge" concentrated at each point. These Taubes' solutions can be interpreted as a nonlinear superposition of single instanton solutions. The non-minimal solutions in our construction admit a similar interpretation as a superposition of instantons and anti-instantons glued to form a "necklace" along the closed geodesic.

The previous theorem can be applied to concrete 4-manifolds, for example, $S^{1} \times S^{3}$ and $S^{2} \times S^{2}$ with the product metrics (see [27]).

This article is organized as follows. In Section 1, we recall Morse theory and provide several examples. Section 2 describes the basic features of YangMills functional. In Section 3-4, by generalizing the Taubes' approach in [22], we introduce the techniques which are used to find the non-minimal solutions to the Yang-Mills equations (cf. [27]). Section 5 gives some remarks, in particular, we consider the existence of non-minimal solutions to the Yang-Mills equations on the round 4-sphere $S^{4}$.

Before turning to a detailed description, acknowledgments are due: The approach in this article was suggested by Clifford Taubes (Actually, this approach was a joint work of Taubes and the author [27].) and inspired by Wente's [28] solution of the Hopf conjecture. Further acknowledgments to the Mathematics Department of the Duke University and Mark Stern for their generosity, kindness and hospitality, where much of this work was carried out.

\section{Morse Theory}

In geometric analysis and mathematical physics we face many elliptic partial differential equations. One asks an important question: Can one rigorously establish the existence of solutions to these equations. 
A strategy is to translate the problem of finding the solutions to the partial differential equations into a problem in the calculus of variations.

Morse theory proposes to relate the critical points of the functional to the topology of the function space. If the functional is nice (in a technical sense) then the function space being topologically complicated will imply that the functional has many critical points (i.e. solutions to the variational equations).

It is perhaps useful to look at a finite dimensional example first [21].

EXAmple 1.1. Let $T^{2}$ be the torus $S^{1} \times S^{1}$ and let $f: T^{2} \rightarrow[0,1]$ be a $C^{2}$ function on $T^{2}$.

Claim: Any such $f$ has at least three critical points.

The maxima and minima of $f$ are of course the critical points. If the minimum is not attained at a unique point then there are already at least three points. Hence we can assume that $f^{-1}(0)=\{p\}$ is just one point.

To find the third critical point in this case, one can use the uncontractible loops on $T^{2}$. Let $\wedge=$ continuous loops on $T^{2}$, starting and ending at $p$ and not contractible on $\left.T^{2}\right\}$. One can apply the min-max method to $\wedge$, showing that

$$
C=\min _{\lambda \in \wedge} \max _{s \in \lambda} f(\lambda(s))
$$

is a critical value that is attained at a critical point with index 1 , so is neither a maximum nor a minimum. For details, see [21].

The relevant lessons from this example are as follows: First, one requires a non-trivial topology to have a good a priori reason to have extra, non-minimal critical points. Second, one requires a compactness to deduce that the min-max sequences actually converge.

Most functionals arising in the geometric analysis and mathematical physics are defined on the infinite dimensional Banach spaces - which are non-compact. There are the standard conditions on a functional on a Banach manifold which, when satisfied, allow one to apply the min-max or Morse theory methods. The most well known of these conditions is the Palais-Smale "Condition $C$ ": A $C^{2}$ function $f: \mathscr{B} \rightarrow \mathscr{R}$ satisfies the Palais-Smale condition on $f^{-1}([a, b])$ if any sequence $\left\{x_{n}\right\}$ such that $a \leq f\left(x_{n}\right) \leq b$ and $\nabla f\left(x_{n}\right) \rightarrow 0$ is precompact.

Unfortunately, the standard conditions do not apply to many interesting functionals. In particular, the Yang-Mills functional does not satisfy the PalaisSmale condition. But the failure of Palais-Smale condition is not the final word for many variational problems. To develop a strategy when Condition $C$ fails, it 
is instructive to consider what can happen on some simple non-compact, finite dimensional manifolds. Now let us consider the following example [3].

EXAMPLE 1.2.

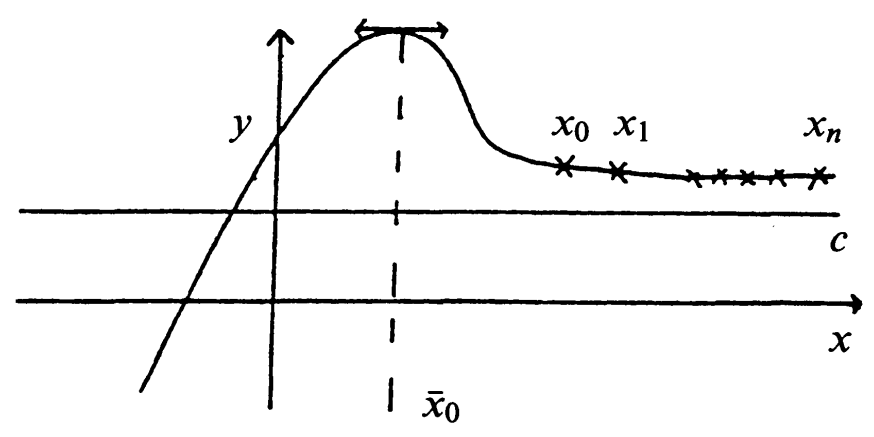

This drawing displays a sequence $\left\{x_{n}\right\}$ (a "critical point at infinity") such that $\nabla f\left(x_{n}\right) \rightarrow 0$ and $f\left(x_{n}\right) \rightarrow c$ which does not have any convergent subsequence. For small $\varepsilon>0$, the set $f^{c+\varepsilon} \triangleq\{x \mid f(x) \leq c+\varepsilon\}$ is not connected while $f^{c-\varepsilon} \triangleq\{x \mid f(x) \leq c-\varepsilon\}$ is connected. Thus this "critical point at infinity" induces a difference of topology in the level sets of $f$.

If one wants to use a global argument to prove the existence of the true critical point $\bar{x}_{0}$, then one should take into account this "critical point at infinity", which plays the role of a minimum.

The lesson to be learned is that when faced with a variational problem for which Condition $C$ fails, one should: (1) find the "relevant non-compact ends" of Banach space (i.e. "critical points at infinity"); (2) analyze the function on the "relevant ends".

The function in Example 1.2 is defined on a contractible space (namely $\mathscr{R}$ ). For the variational problems on a general Banach manifold $\mathscr{X}$ one must understand which topological features of $\mathscr{X}$ are represented by actual critical points of $f$ and which are accounted for by the non-compact ends.

The "relevant ends" (both compact and non-compact) of $f$ can be defined as follows:

$$
\begin{gathered}
\text { Crit. }(N, \delta)=\{x \in \mathscr{X} \mid N-\delta<f(x)<N+\delta, \quad \text { and }\|\nabla f x\|<\delta\}, \\
\text { Crit. }(N, \delta)^{-}=\{x \in \text { Crit. }(N, \delta) \mid f(x)<N\} .
\end{gathered}
$$

When $\delta>\delta^{\prime}>0$ are given, there is a natural inclusion pairs:

$$
i:\left(\operatorname{Crit} .\left(N, \delta^{\prime}\right), \operatorname{Crit} .\left(N, \delta^{\prime}\right)^{-}\right) \longrightarrow\left(\operatorname{Crit} .(N, \delta), \operatorname{Crit} .(N, \delta)^{-}\right) .
$$


It is the effect of the map $i$ on the relevant homotopy groups of pairs which determines whether Crit. $(N, \delta)$ contributes to the Morse theory of $f$ on $\mathscr{X}$.

Intuitively, the "relevant ends" of $f$ consist of approximate solutions to the Euler-Lagrange equations of $f$. One can then hope that by a small perturbation, one can find the exact solutions to the Euler-Lagrange equations.

Finally, let us briefly recall the Taubes' approach to the self-dual connections over the generic 4-manifolds [22].

EXAMPLE 1.3. Let $M$ be a compact, oriented, Riemannian 4-manifold. By the gluing operation, one may graft the basic instantons over $S^{4}$ into the trivial connection over $M$ at distinct points in $M$ to get an approximate solution space for the self-dual equations on $M$. Also one has a parameter space $N$. For any $y \in N$, one has an approximate solution $A(y)$. The Taubes' approach to the selfdual equations on $M$ is that by the small perturbation, one may solve the nonlinear elliptic equations to get an exact self-dual connection over $M$. But the linearization of the self-dual equations on $M$ at an approximate solution is not strongly elliptic, there are the obstructions to solving the self-dual equations which come from the small eigenspace of the Hessian of Yang-Mills functional at this approximate solution that arises from the negative part of the intersection form on $M$. In order to overcome this difficulty, Taubes considered the expansion of Yang-Mills functional restricted on the approximate solution space in parameters. Taubes proved that if the degree of the bundle constructed by the gluing operation was large enough, the parameters account for all the small eigenvectors. Hence one may solve the self-dual equations in the direction of the complement of the small eigenspace, then move the positions of parameters to find an exact self-dual connection over $M$. For details, refer to [22].

The lesson of this example: To construct a Morse theory with a functional, $f$, on a non-compact Banach manifold, $\mathscr{X}$, an appropriate strategy is to 1) find the set $\operatorname{Crit}(N, \delta)$ in the Banach manifold as defined above. 2) Restrict the functional to these sets and study the effect of the map $i$ on the relative topology of the pairs of space. 3) Determine the obstructions to finding the "true critical points."

\section{Yang-Mills Functional}

The Yang-Mills equations were introduced by theoretical physicists. In the past decade these equations became important in mathematics. Simon Donaldson obtained beautiful and spectacular results concerning the differential structures 
on the four-manifolds by using the moduli spaces of the self-dual solutions to the Yang-Mills equations on the compact 4-manifolds [[9], [10]). Atiyah-HitchinSinger [1] and Freed-Uhlenbeck [10] gave good simple introduction to the YangMills equations, one can find useful materials in these references.

Let $P$ be a principal $S U(2)$-bundle over a compact, oriented 4-manifold, $M$, with a Riemannian metric $g$. Let $\mathscr{C}(P)$ be the connection space which is an affine space. Fix $A_{0} \in \mathscr{C}(P), \mathscr{C}(P)=A_{0}+\Omega^{1}(A d P)$, here $A d P$ is the adjoint bundle.

Consider the Yang-Mills functional (action functional) on $\mathscr{C}(P)$ :

$$
Y M(A)=\frac{1}{2} \int_{M}\left|F_{A}\right|^{2} .
$$

Where, $F_{A}$ is the curvature of $A, F_{A} \in \Omega^{2}(A d P)$. The norm [2.1) is induced from the Riemannian metric on $T M$ and the Killing form on the Lie algebra of $S U(2)$. The Yang-Mills functional has a topological lower bound

$$
Y M(A)=\frac{1}{2} \int_{m}\left|F_{A}\right|^{2} \geq 8 \pi^{2}\left|C_{2}(P)\right| .
$$

Where, $C_{2}(A)$ is the second Chern class of $P$.

The variational equations of $Y M$ is

$$
\nabla Y M_{A}(\cdot)=0
$$

or

$$
D_{A}^{*} F_{A}=0 .
$$

Here $D_{A}$ is the covariant exterior differential associated to $A, D_{A}^{*}$ is the formal adjoint operator of $D_{A}$. Equation (2.4) is the second order, non-linear partial differential equation.

A connection $A$ is an absolute minimal solution to (2.4) if and only if $F_{A}= \pm * F_{A}$ where $*$ is the Hodge star operator and the sign is + if $C_{2}(P)<0$ and - if $C_{2}(P)>0$. The equations $F_{A}= \pm * F_{A}$ are called the self-dual or the anti-self-dual equations. These equations are the first order P.D.E. like as the Cauchy-Riemann equations on the two dimensional surfaces.

In terms of the algebraic method, one is easily to construct the basic self-dual or the basic anti-self-dual solutions over $S^{4}$. These solutions are called the instantons or the anti-instantons. Using the stereographic projection $S^{4} \backslash\{$ south pole $\} \rightarrow \mathscr{R}^{4}$, identify $\mathscr{R}^{4} \simeq \mathscr{H}$ quaternions. One has the following explicit formulas: 
On $\mathscr{R}^{4}$, define

$$
\left\{\begin{array}{l}
U_{1}=\left\{x \in \mathscr{R}^{4}|| x \mid<1\right\} \\
U_{2}=\mathscr{R}^{4} \backslash\{O\} .
\end{array}\right.
$$

The basic instantons are expressed as follows:

$$
W_{\lambda_{+}}=\left(W_{\lambda_{+}}^{1}, W_{\lambda_{+}}^{2}\right)=\left(\operatorname{Im} \frac{x d \bar{x}}{\lambda^{2}+|x|^{2}}, \operatorname{Im} \frac{\lambda^{2} \bar{x} d x}{|x|^{2}\left(\lambda^{2}+|x|^{2}\right)}\right)
$$

with the transition function

$$
\left\{\begin{array}{l}
g_{12}^{+}: U_{1} \cap U_{2} \longrightarrow S U(2) \\
g_{12}^{+}(x)=\frac{x}{|x|}
\end{array}\right.
$$

Where, $\lambda$ is the scaling parameter. The curvature of this connection is given by

$$
F_{\lambda_{+}}=\left(F_{\lambda_{+}}^{1}, F_{\lambda_{+}}^{2}\right)=\left(\frac{\lambda^{2} d x \wedge d \bar{x}}{\left(\lambda^{2}+|x|^{2}\right)^{2}}, \frac{\lambda^{2} \bar{x} d x \wedge d \bar{x} x}{|x|\left(\lambda^{2}+|x|^{2}\right)^{2}|x|}\right) .
$$

Similarly, the basic anti-instantons are expressed as follows:

$$
W_{\lambda_{-}}=\left(W_{\lambda_{-}}^{1}, W_{\lambda_{-}}^{2}\right)=\left(\operatorname{Im} \frac{\bar{x} d x}{\lambda^{2}+|x|^{2}}, \operatorname{Im} \frac{\lambda^{2} x d \bar{x}}{|x|^{2}\left(\lambda^{2}+|x|^{2}\right)}\right)
$$

with the transition function

$$
\left\{\begin{array}{l}
g_{12}^{-}: U_{1} \cap U_{2} \longrightarrow S U(2) \\
g_{12}^{-}(x)=\frac{x}{|x|}
\end{array}\right.
$$

The curvature of $W_{\lambda_{-}}$is given by

$$
F_{\lambda_{-}}=\left(F_{\lambda_{-}}^{1}, F_{\lambda_{-}}^{2}\right)=\left(\frac{\lambda^{2} d \bar{x} \wedge d x}{\left(\lambda^{2}+|x|^{2}\right)^{2}}, \frac{\lambda^{2} x d \bar{x} \wedge d x \bar{x}}{|x|\left(\lambda^{2}+|x|^{2}\right)^{2}|x|}\right) .
$$

An important problem in the analytic aspect of the Yang-Mills theory is to find the general solutions to the Yang-Mills equations. One of the difficulties is that the Yang-Mills equations are not strong elliptic P.D.E.. Because the YangMills equations are invariant under the action of the gauge transformation group $A u t P$ (this is the inner automorphism group of $P$ which can be identified with the group of the smooth cross-sections of a group bundle $P \times_{A d} S U(2)$, the 
Hessian of Yang-Mills functional has an infinite dimensional null space: If $A$ is a solution of Yang-Mills equations then $\nabla^{2} Y M_{A}(a, \cdot)=0$ for any $a=D_{A} \phi$ where $\phi \in \Omega^{0}(A d P)$. Indeed $\Omega^{0}(A d P)$ can be viewed as the infinitesimal gauge group or the gauge algebra. By the direct calculation, the Hessian of. Yang-Mills functional may be written as

$$
\nabla^{2} Y M_{A}(a, b)=\int_{M}\left(D_{A} a, D_{A} b+\left(F_{A}, a \wedge b+b \wedge a\right) .\right.
$$

This pathology can be remedied by considering $Y M(\cdot)$ as a functional on the quotient space $\mathscr{B}(P) \triangleq \mathscr{C}(P) / A u t P$. Since $M$ is a compact 4-manifold, one can define the $L_{k}^{2}$-Sobolev structure $(k \geq 3)$ on $\mathscr{B}(P)$ which is viewed as a Hilbert manifold as in [23]. When restricted to the tangent space of an orbit $[A] \in \mathscr{B}(P)$, $\nabla^{2} Y M_{A}(\cdot, \cdot)$ defines a bounded, elliptic bilinear form. We would like to point out here that the quotient space $\mathscr{B}(P)$ is not quite Banach manifold since when $A$ is an reducible connection then the tangent space to $[A] \in \mathscr{B}(P)$ is an infinite dimensional cone. Recall the reducible connection, we say $[A] \in \mathscr{C}(P)$ is reducible if the principal $S U(2)$-bundle $P \cong \lambda_{1} \oplus \lambda_{2}$ and the covariant exterior differential $D_{A}=d_{1} \oplus d_{2}$ are both decomposable. Here $\lambda_{2}=\lambda_{1}^{-1}$, such splitting corresponding to the singular points on $\mathscr{B}(P)$. For details of the above, refer to [8], [10].

Actually, the main difficulty in solving the Yang-Mills equations lies in the failure of Palais-Smale conditions for the Yang-Mills functional. K. K. Uhlenbeck established the weak compactness theorem for the Yang-Mills functional [[14], [23], [25]). The weak compactness of a good sequence (in technical sense) is discussed in Proposition 2.1, below. This proposition is in many respects analogous to the existence theorem for the harmonic maps from $S^{2}$ that are derived by Sacks and Uhlenbeck [19].

Proposition 2.1 (cf. [23]). Let $M$ be a compact, oriented, Riemannian 4manifold. Let $P \rightarrow M$ be a principal $S U(2)$-bundle with the degree $n \geq 0$. Let $\left\{\left[A_{i}\right]\right\} \subset \mathscr{B}(P)$ be a good sequence for which $\lim _{i \rightarrow \infty} Y M\left(A_{i}\right) \rightarrow Y M_{\infty}$. There exists a subsequence of $\left\{\left[A_{i}\right]\right\}$, also denoted $\left\{\left[A_{i}\right]\right\}$, and a finite set of the pairs $\left\{\left(P_{\alpha}, A_{\alpha}\right)\right\}_{\alpha=0}^{m}$, where $P_{0} \rightarrow M$ is a principal $S U(2)$-bundle and $A_{0}$ is a smooth connection on $P_{0}$ which is a solution to the Yang-Mills equations on $M$. While for $\alpha>0$, each $P_{\alpha} \rightarrow S^{4}$ is a principal $S U(2)$-bundle and $A_{\alpha}$ is a smooth connection on $P_{\alpha}$ which is a solution to the Yang-Mills equations on $S^{4}$ for the standard metric on $T^{*} S^{4}$. These data have the following properties:

(1) $\left\{\left[A_{i}\right]\right\}$ converges strongly in $L_{1, l o c}^{2}$ of $M \backslash\{$ finite set $\}$ to $\left[A_{0}\right]$;

(2) For $\alpha>0, A_{\alpha}$ is not flat; 
(3) $\sum_{\alpha=0}^{m} Y M\left(A_{\alpha}\right)=Y M_{\infty}$;

(4) $\sum_{\alpha=0}^{m} k\left(P_{\alpha}\right)=n$.

Here the good sequence of connections, $\left\{A_{i}\right\}$, in $\mathscr{B}(P)$ means that $\left\{Y M\left(A_{i}\right)\right\}$ is bounded and $\lim _{i \rightarrow \infty}\left\|\nabla Y M_{A_{i}}(\cdot)\right\|_{*} \rightarrow 0$.

Briefly this proposition says that any good sequence of connections which goes to infinity has a subsequence which, outside some points $\left\{p_{j}\right\}_{j=1}^{m} \subset$ $M, m \leq n$, converges to a true solution of Yang-Mills equations (may be a trivial connection) uniformly on the compact subsets and whose a portion of the energy becomes concentrated in smaller and smaller neighborhoods of $\left\{p_{j}\right\}_{j=1}^{m}$. In fact, by choosing an appropriate sequence of the blow-up of the normal coordinates at $\left\{p_{j}\right\}_{j=1}^{m}$ over these smaller and smaller neighborhoods the connections can be seen to converge to the Yang-Mills connections over $S^{4}$. At the last moment (i.e. in the limit) the bundle $P$ becomes the connected sum of $\left\{P_{\alpha}\right\}_{\alpha=0}^{m}$, and the standard 4-spheres break off at $\left\{p_{j}\right\}_{j=1}^{m}$, carrying with them the partial energy and topology of the old connection. It is possible that there are two or more 4-spheres bubbling off at one point $p_{j}$.

In subsequent sections, we shall devote to finding the "end sets" of YangMills functional (i.e. approximate solution space), studying the effect of YangMills functional restricted to these sets and finding the non-minimal solutions of Yang-Mills equations in terms of the perturbation method.

\section{The Gluing Construction}

Naive Morse theory will not work for the Yang-Mills functional on the quotient space $\mathscr{B}(P)$ due to the Uhlenbeck's weak compactness theorem. According to the lessons learned from the examples in Section 1, one should find the "end sets" ("critical points at infinity") in $\mathscr{B}(P)$. The weak compactness theorem provides the crucial tool for describing these ends. Taubes [24] showed that the weak-compactness can be reversed by "gluing in" in instantons and anti-instantons over $S^{4}$. Indeed, by the gluing operation one is able to graft the instantons and anti-instantons over $S^{4}$ onto a background connection over the generic manifold, $M$, at distinct points $M$, and to construct the "end sets".

Using these "end sets", Taubes constructed the self-dual and anti-self connections on the compact oriented 4-manifolds and explained how the Morse theory for the Yang-Mills functional can be recovered by examining the restriction of the functional to these sets. Actually, the "end set" is a countable 
set of finite dimensional, non-compact varieties. For details, refer to [22], [24].

There is some trouble in extending the Taubes' approach to the problem of finding the non-minimal solutions of Yang-Mills equations. To explain this, consider the following simple example.

EXAMPLE 3.1. By using the gluing operation, graft an instanton with the scaling parameter $\lambda_{1}$, the gauge $g_{1} \in S U(2)$ and an anti-instanton with the scaling parameter $\lambda_{2}$, the gauge $g_{2} \in S U(2)$ onto the trivial connection over $S^{4}$ at points $\left\{p_{1}\right\}$ and $\left\{p_{2}\right\}$, respectively. Hence one has a family of approximate solutions which depend on the parameters $\left\{\lambda_{1}, g_{1}, p_{1}, \lambda_{2}, g_{2}, p_{2}\right\}$. Consider the Taylor's expansion of the action functional in the parameters [26]:

$$
\begin{aligned}
Y M\left(A\left(\lambda_{1}, g_{1}, p_{1}, \lambda_{2}, g_{2}, p_{2}\right)\right)= & 16 \pi^{2}-\frac{Q \lambda_{1}^{2} \lambda_{2}^{2}}{\text { dist. }\left(p_{1}, p_{2}\right)^{4}}\left\{\left\langle g_{1} F_{+}(N) g_{1}^{-1}, g_{2} F_{+}(N) g_{2}^{-1}\right\rangle\right. \\
& \left.+\left\langle g_{2} F_{-}(N) g_{2}^{-1}, g_{1} F_{-}(N) g_{1}^{-1}\right\rangle\right\} \\
& + \text { higher order term },
\end{aligned}
$$

where $Q$ is a universal constant and $F_{+}(N), F_{-}(N)$ are the curvatures of the standard instanton and anti-instanton at the north pole.

To explain Example 3.1, recall the Taubes' implicit function theorem [21]. One may solve the non-linear elliptic equations according to the following procedure: First, construct an approximate solution space of the equations. Second, consider the Taylor expansion of the corresponding functional of the equations in the parameters (that is, the functional is restricted on the approximate solution space). Third, find the non-degenerate critical points of the first order term of the functional restricted on the approximate solution space. Finally, by the small perturbation get an exact solution to the equations (Here, use the implicit function theorem to kill the higher order terms of the functional.)

Now turn to Example 3.1. If one wants to find the critical points near those almost Yang-Mills connections that were constructed in Example 3.1, one should find the critical points of the following the first order term of Yang-Mills functional restricted on the approximate solution space.

$$
\frac{Q \lambda_{1}^{2} \lambda_{2}^{2}}{\text { dist. }\left(p_{1}, p_{2}\right)^{4}} \cdot\left\{\left\langle g_{1} F_{+}(N) g_{1}^{-1}, g_{2+}^{F}(N) g_{2}^{-1}\right\rangle+\left\langle g_{1} F_{-}(N) g_{1}^{-1}, g_{2} F_{-}(N) g_{2}^{-1}\right\rangle\right\} \text {. }
$$


By the direct calculation, it is not hard to see that we must let dist. $\left(p_{1}, p_{2}\right) \rightarrow \pi$, and $\lambda_{1}, \lambda_{2} \rightarrow 0$. Therefore finding the non-minimal solutions in the preceding construction will be bubbling off two spheres at the north and south poles respectively, this means that the instanton and anti-instanton will be blown up. Hence, the Taubes' implicit function theorem does not work here.

On the other hand, Parker [12] showed how, by changing the metric on $S^{4}$ amount, one can form a non-minimal Yang-Mills connection with degree zero and with energy arbitrarily close to two instanton units. Parker's solution consists of a very small instanton and a very small anti-instanton, centered at the antipodal points. If the metric were round, as before discussing, both the instanton and anti-instanton would bubble off under the gradient flow. However, with the slightly altered metric, the instanton and anti-instanton become balance. Hence, Parker in effect turned the "end point" into a critical point.

According to the example discussed above, one can not directly use the Taubes' approach to find the non-minimal solutions. The situation is more complicated. One requires that the approximate solutions have nice properties. Hence, one must make small change to look for suitably a balance condition for the approximate solutions. We now describe our construction in some generality [27]. This construction was inspired by Wente's [28] solution of the Hopf conjecture.

Let $P_{0} \rightarrow M$ be a background principal $S U(2)$ bundle over $M$, let $A_{0}$ be a smooth background connection on $P_{0}$. Now, let $C$ be a simple closed geodesic on $M$ (Without loss of generality, assume that the length of $C$ is equal to $2 \pi$ exactly.). Fix a tubular neighborhood, $V_{0}$, of $C$. Let $\left\{s, y^{\alpha}\right\}_{\alpha=1}^{3}$ be a coordinate system on $V_{0}$ with $s: V_{0} \rightarrow[0,2 \pi]$ restricting to $C$ as the arclength. Require $\left.y^{\alpha}\right|_{C}=0$ and $\left\{\partial / \partial s, \partial / \partial y^{\alpha}\right\}_{\alpha=1}^{3}$ orthonormal on $C$.

Choose $2 k$ points $\left\{q_{1}, \ldots, q_{2 k}\right\}$ in $V_{0}$ such that

$$
\frac{5 \pi}{4 k}>s\left(q_{i+1}\right)-s\left(q_{i}\right)>\frac{3 \pi}{4 k} .
$$

For sake of simplicity, set $d=\pi / k$. Over each $q_{i}$, pick up $f_{i} \in F_{M} \mid q_{i}$ (Here, $F_{M}$ is the frame bundle of $T M$.$) . We have the Gaussian coordinate system \phi_{f_{i}}: U\left(q_{i}\right) \rightarrow$ $B_{\rho} \subset \mathscr{R}^{4}$. Where $\rho$ is the injectivity radius of $M$, and require $d<\rho$. Choose $r \in(d, \rho)$ and for each $i$, choose $\lambda_{i} \in(0, r / 2), g_{i} \in S U(2)$. Together, the data $C$ and $\left(\lambda_{i}, f_{i}, g_{i}\right)_{i=1}^{2 k}$ gives our parameters for gluing. 
Set

$$
\left\{\begin{array}{l}
V_{i}=\left\{x \mid 0<\text { dist. }\left(x, q_{i}\right)<r\right\} \\
U_{i}=\left\{x \mid \text { dist. }\left(x, q_{i}\right)<\lambda_{i}\right\}
\end{array}\right.
$$

Reintroduce the connection $A_{0}$ and fix a gauge along $V_{0}$ in which $\left.A_{0}\right|_{v_{0}}=\Gamma+a$ where

$$
\begin{aligned}
& \left.\langle 1\rangle \quad \frac{\partial}{\partial_{s}}\right\rfloor a=\sigma \quad \text { obeys } \\
& \left.\langle 2\rangle \quad \frac{\partial}{\partial_{s}}\right\rfloor \nabla_{\Gamma} \sigma=0, \\
& \left.\left.\langle 3\rangle \quad a\right|_{C}=\left.\sigma\right|_{C} d s \text { and } y^{\alpha} \frac{\partial}{\partial y^{\alpha}}\right\rfloor a=0 .
\end{aligned}
$$

Here, the gauge is unique up to $\sigma \rightarrow g \sigma g^{-1}$ for $g \in S U(2)$. It actually depends only on the holonomy of the connection $A_{0}$ around $C$. In fact $\left.P_{0}\right|_{V_{0}}$ can be trivialized over open domain $V_{0}$, hence $\left.A_{0}\right|_{V_{0}}$ can be written as $\left.A_{0}\right|_{V_{0}}=\Gamma+a$ where $a$ satisfies the conditions (3.2) by choosing suitable gauge (i.e. solving the ordinary differential equations along $C$ ).

We shall glue the instantons and anti-instantons onto $A_{0}$ along the geodesic $C$ and for this, we require the bump function $\beta$. This is a smooth function on $\mathscr{R}$, $0 \leq \beta \leq 1$ with $\beta(t)=1$ if $t<1$ and $\beta(t)=0$ as $t>2$.

With this all understood, set

$$
A=\left\{\begin{array}{l}
A_{0} \text { over } M \backslash V_{0}, \\
\Gamma+\sum_{i=1}^{2 k} \beta_{r}\left(x, q_{i}\right) \phi_{f_{i}}^{*} g_{i} W_{i}^{2} g_{i}^{-1}+a \text { over } V_{0} \backslash \bigcup_{i=1}^{2 k} V_{i}, \\
\Gamma+h_{i}\left[\phi_{f_{i}}^{*} g_{i} W_{i}^{2} g_{i}^{-1}+\left(1-\beta_{\lambda_{i}}\left(x, q_{i}\right)\right.\right. \\
\left.\quad \cdot\left(a+\sum_{j \neq i} \beta_{r}\left(x, q_{j}\right) \phi_{f_{j}}^{*} g_{j} W_{j}^{2} g_{j}^{-1}\right)\right] h_{i}^{-1}+h_{i} d h_{i}^{-1} \text { over } V_{i} \backslash U_{i}, \\
\phi_{f_{i}}^{*} g_{i} W_{i}^{1} g_{i}^{-1} \text { over } U_{i} .
\end{array}\right.
$$

Here, $\left(W_{i}^{1}, W_{i}^{2}\right)=\left(W_{\lambda_{i}+}^{1}, W_{\lambda_{i}+}^{2}\right)$ as $i=$ odd and $\left(W_{i}^{1}, W_{i}^{2}\right)=\left(W_{\lambda_{i}-}^{1}, W_{\lambda_{i}-}^{2}\right)$ as $i=$ even. The gauge transformation $h_{i}$ is given by requiring that $h_{i}\left(q_{i}\right)=1$ and

$$
\alpha_{i}=h_{i}\left(a+\sum_{j \neq i} \beta_{r}\left(x, q_{j}\right) \phi_{f_{j}}^{*} g_{j} W_{j}^{2} g_{j}^{-1}\right) h_{i}^{-1}+h_{i} d h_{i}^{-1}
$$


obeys

$$
\alpha_{i}\left(q_{i}\right)=0 \quad \text { and } \quad \frac{\partial}{\partial\left|x-q_{i}\right|} \mid \alpha_{i}=0 .
$$

In fact, $h_{i}$ is the polar gauge transformation which is given by solving the ordinary differential equations. Usually it is easy to do computation in polar gauge (cf. [23], [24], [25] and [27]). So, we get a parameter space

$$
N_{1} \subset(d, \rho) \times\left. P_{0}\right|_{q_{0}} \times \prod_{i=1}^{2 k}\left(\left(0, \frac{r}{2}\right) \times F_{M} \times S U(2)\right),
$$

and a map

$$
\left\{\begin{aligned}
& \Psi: N_{1} \rightarrow \mathscr{B}(P) \\
& N_{1} \ni y \mapsto(P, A(y))
\end{aligned}\right.
$$

with $-C_{2}\left((A(y))=-C_{2}\left(A_{0}\right)\right.$ as $\lambda_{i}$ sufficiently small.

However, the map $\Psi$ is not an injective map as it is invariant under the certain symmetry group which acts on $N_{1}$. This invariance of the map $\Psi$ is due to the special structure group $S O(4)$ of the frame bundle $F_{M}$. It is well known that structure group $S O(4) \cong S U(2) \times_{\{ \pm\}} S U(2)$. Hence $\left.A d P\right|_{q_{0}} \cong P_{ \pm}$ $\left.\wedge^{2} T^{*} M\right|_{q_{0}}$. Thus the parameter space may be reduced. Define

$$
\begin{aligned}
N_{2} \subset(d, \rho) & \times\left. P_{0}\right|_{q_{0}} \times_{S U(2)} \prod_{i=1}^{2 k}\left(\left(0, \frac{r}{2}\right) \times P_{ \pm} \wedge^{2} T^{*} M \times_{S U(2)} S U(2)\right) \\
& \simeq(d, \rho) \times \prod_{i=1}^{2 k}\left(\left(0, \frac{r}{2}\right) \times P_{ \pm} \wedge^{2} T^{*} M\right) / \Gamma_{A_{0}}
\end{aligned}
$$

Hence, \pm 1 depend on the parity of $i$, and $\Gamma_{A_{0}}$ is the centralizer of gauge group $\operatorname{Aut}\left(A_{0}\right)$. Hence, the map factors through $N_{2}$.

Therefore we obtain the "end sets". If the background connection $A_{0}$ is a solution to the Yang-Mills equations, then $\Psi\left(N_{2}\right) \subset \mathscr{B}(P)$ is an approximate solution space. In the remainder of this article, we always require that $A_{0}$ is a solution of Yang-Mills equations.

We now study the interaction between the mixed instantons and the background connection. Set $r=d^{3 / 5}, \lambda_{i}=s_{i} d^{2}$. For any $y \in N_{2}$, expand $Y M(A(y))$ in the parameter $d$.

Proposition 3.1 (cf. [27]). If the Yang-Mills functional is restricted to the parameter space, one has 


$$
\begin{aligned}
& Y M(A(y))=\frac{1}{2} \int_{m}\left|F_{A}(y)\right|^{2} \\
& =Y M\left(A_{0}\right)+16 \pi^{2} k \\
& +\sum_{i=\mathrm{odd}}-\frac{\omega\left(s^{3}\right)}{2} s_{i}^{2} d^{4}\left\langle P_{-} F_{A_{0}}\left(q_{i}\right), \phi_{f_{i}}^{*} g_{i} F_{-}(N) g_{i}^{-1}\right\rangle \\
& +\sum_{j=\text { even }}-\frac{\omega\left(s^{3}\right)}{2} s_{j}^{2} d^{4}\left\langle P_{+} F_{A_{0}}\left(q_{j}\right), \phi_{f_{j}}^{*} g_{j} F_{+}(N) g_{i}^{-1}\right\rangle \\
& +\sum_{i=\text { odd }} \sum_{\substack{j=\text { even } \\
j=[i \pm(2 l+1)] \bmod 2 k \\
0 \leq l \leq\left[1 / 2 d^{-2 / 5}\right]-1}}-Q \frac{\omega\left(S^{3}\right)}{2} s_{i}^{2} s_{j}^{2} d^{8} / \operatorname{dist} .\left(q_{j}, q_{i}\right)^{4} \\
& \cdot\left\langle\phi_{f_{i}}^{*} g_{i} F_{-}(N) g_{i}^{-1}, \phi_{f_{j}}^{*} g_{j} F_{-}(N) g_{j}^{-1}\right\rangle \\
& +\sum_{j=\text { even }} \sum_{\substack{i=\text { odd } \\
i=[j \pm(2 l+1)] \bmod 2 k \\
0 \leq l \leq\left[1 / 2 d^{-2 / 5}\right]-1}}-Q \frac{\omega\left(S^{3}\right)}{2} s_{j}^{2} s_{i}^{2} d^{8} / \text { dist. }\left(q_{j}, q_{i}\right)^{4} \\
& \cdot\left\langle\phi_{f_{j}}^{*} g_{j} F_{+}(N) g_{j}^{-1}, \phi_{f_{i}}^{*} g_{i} F_{+}(N) g_{i}^{-1}\right\rangle \\
& +\sum_{i=1}^{2 k}\left\{s_{i}^{2} d^{5(1 / 5)}\left(C_{1}+C_{2}|\ln d|+\text { higher order terms }\right\}\right.
\end{aligned}
$$

where $Q$ is a positive constant.

In terms of the Taylor's expansion above, one can give a priori estimate for the dual norm, $\left\|\nabla Y M_{A(y)}(\cdot)\right\|_{A(y)}^{*}$, of the first variation of Yang-Mills functional

$$
\left\|\nabla Y M_{A(y)}(\cdot)\right\|_{A(y)}^{*}, \leq C_{3}\left\{\sum_{i=1}^{2 k} s_{i}^{2} d^{5(1 / 5)}\right\} .
$$

We now give remarks about the gluing operation and expanding the Yang-Mills functional in the power series in parameters. There is a physics intuition behind the expansion of Yang-Mills functional. In physics, the connection is supposed to describe the force field due to certain the pseudo-particles. For a physicist, the Yang-Mills functional measures the energy that is contained in the force field. The connect-sum construction (the gluing operation) can be interpreted in a heuristic sense as the addition of the specific, extra pseudo-particles to a system of pseudo-particles (each pseudo-particle contributes a unit charge to the instanton number). 
If the new pseudo-particle can be added to make the net force attractive (or repulsive), the normalized energy, the first order term of the expansion of YangMills functional, will decrease (increase). Hence, the first order term of the functional is viewed as the interaction between the instantons and antiinstantons. This kind of the interaction phenomena of the "mixed pseudoparticles" has been used by Taubes [23] for the Yang-Mills equations on $S^{4}$, it has allowed him to prove that the Yang-Mills moduli spaces of $S U(2)$ (or $S U(3))$ connections are path-connected spaces. It has been also considered by Bahri-Coron (cf. [3], [5]), they have used it to prove that the existence theorem for the Yamabe equation on the certain domain in $\mathscr{R}^{n}$.

Similarly, a simple calculation verifies the Yang-Mills-Higgs functional: Monopoles attract anti-monopoles at large separation. By the min-max technique, Taubes [21] rigorously established the existence of non-minimal solutions of Yang-Mills-Higgs functional.

For the Yang-Mills functional, the situation is more complicated. Let us look at the forces of the mixed pseudo-particles in our construction. The first order term of the functional smoothly depends on the parameters $\left\{s_{i}, g_{i}, q_{i}\right\}_{1 \leq i \leq 2 k}\left(\operatorname{dim} . N_{2}=16 k\right)$. The important fact is that for the first order term of Yang-Mills functional in the preceding Taylor expansion, there exists a saddle point on the parameter space $N_{2}$.

The conclusion here is that the unstable solutions of the higher energy should not be unexpected.

\section{The Global Setting}

The heading of this section asks an important question: Can one rigorously establish the existence of non-minimal solutions to the Yang-Mills equations. The proof for the cases $S^{2} \times S^{2}$ and $S^{1} \times S^{3}$ were developed by the author [27]; $S^{4}$ and $S^{1} \times S^{3}$ were proved by L. \& R. Sibner and K. Uhlenbeck [20], T. Parker [12], and L. Sadun and J. Segert [16], [17].

The strategy is to use the perturbation method in which Taubes constructed many self-dual or anti-self-dual connections over the generic 4-manifolds. By this, one means the following: Suppose $A(y), y \in N_{2}$ is a approximate solution and $a(y) \in \Omega^{1}(A d P)$, then $A(y)+a(y)$ is a solution to the Yang-Mills equations if and only if the energy, $Y M$, is stationary with respect to all the infinitesimal variation of the fields. That is, if and only if

for all $\varphi \in \mathbf{\Omega}^{1}(\operatorname{AdP})$.

$$
\left.\frac{d}{d t} Y M(A(y)+a(y)+t \varphi)\right|_{t=0}=0
$$


Hence, we must solve the equation below:

$$
\nabla Y M_{A(y)+a}(\cdot)=0, \quad \text { for } y \in N_{2} .
$$

But the equation given above is not strong elliptic. By the Taylor expansion, the small eigenspace of the Hessian, $\nabla^{2} Y M_{A(y)}(\cdot, \cdot)$, of $Y M$ is the obstruction to solving Equation (4.1). Let us analyze the obstruction to solving Equation (4.1) (i.e the small eigenspace of $\left.\nabla^{2} Y M_{A(y)}(\cdot, \cdot)\right)$. According to the construction of the approximate solutions, $A(y)$ for $y \in N_{2}$, it is reasonable to think that the small eigenvalues of $\nabla^{2} Y M_{A(y)}(\cdot, \cdot)$ come from the small eigenvalues of $\nabla^{2} Y M_{A_{0}}(\cdot, \cdot)$ and $\nabla^{2} Y M_{W_{ \pm}}(\cdot, \cdot)$. For details, refer to [4], [24] and [27].

Henceforth, always take the background connection, $A_{0}$, to be an isolated solution to the Yang-Mills equations, with $\left|P_{ \pm} F_{A_{0}}\right|$ a non-zero constant and $d\left|P_{ \pm} F_{A_{0}}\right|=0$ along the geodesic $C$. Since the background connection $A_{0}$ is an isolated solution, the small eigenspace of $\nabla^{2} Y M_{A(y)}(\cdot, \cdot)$ depends only on the small eigenvalues of $\nabla^{2} Y M_{W_{ \pm}}(\cdot, \cdot)$. By direct calculation, it is not hard to obtain that the small eigenspace of $\nabla^{2} Y M_{A(y)}(\cdot, \cdot)$ is of dimension $16 k$. Hence, our parameters account for all the small eigenvalues of $\nabla^{2} Y M_{A(y)}(\cdot, \cdot)$ $\left(\operatorname{dim} N_{2}=16 k\right)$ (cf. [26]). The small eigenspace of $\nabla^{2} Y M_{A(y)}(\cdot, \cdot)$ at each $y \in N_{2}$ defines a vector bundle $V \rightarrow N_{2}$ as a subspace of $\Psi^{*} T \mathscr{B}$. Here, $\Psi: N_{2} \rightarrow \mathscr{B}$ is an inclusion. It is possible to divide Equation (4.1) into two parts

$$
(I-\pi(A(y)+a, \xi))^{*} D_{A(y)+a}^{*} F_{A(y)+a}=0,
$$

and

$$
\pi(A(y)+a, \xi)^{*} D_{A(y)+a}^{*} F_{A(y)+a}=0 .
$$

Here $\pi(A(y)+a, \xi)$ is a projection onto the small eigenspace of $\nabla^{2} Y M_{A(y)+a}(\cdot, \cdot)$.

Equation (4.2) is a strong elliptic equation. Let $\mathscr{U}_{\xi}(y)$ be the unique solution of Equation (4.2). One defines

$$
f_{\xi}(y)=\pi\left(A(y)+\mathscr{U}_{\xi}(y), \xi\right)^{*} D_{A(y)+\mathscr{U}_{\xi}(y)}^{*} F_{A(y)+\mathscr{U}_{\xi}(y)}
$$

In fact, $f_{\xi}(y)$ is a smooth section on the vector bundle $V \rightarrow N_{2}$. The key step is to find the zero points of $f_{\xi}(y)$. Using the Kuranishi's method (which is just the implicit function theorem), write $f_{\xi}(y)=f_{\xi}^{1}(y)+f_{\xi}^{2}(y)$. Suppose that $f_{\xi}^{1}(\bar{y})=0$ and $\nabla f_{\xi}^{1}(\bar{y})$ is non-degenerate. If $f_{\xi}^{2}(y)$ is the higher order term, by moving the positions of $y=\left\{\left(s_{i}, g_{i}, q_{i}\right)_{i=1}^{2 k}\right\}$ we get $f_{\xi}(\tilde{y})=0$ via the implicit function theorem. Here $\tilde{y}$ near by $\bar{y}$ (cf. [22], [27]). Hence one must find a suitable $f_{\xi}^{1}(y)$ and analyze the properties of $f_{\xi}^{1}(y)$. 
We now recall Proposition 3.1. By the Taylor expansion, the first order term of the functional, $Y M(A(y))$, smoothly depends on the parameters $\left\{\left(s_{i}, q_{i}, g_{i}\right)_{i=1}^{2 k}\right\}$. Let $H$ denote the first order term of the Taylor's expansion of Yang-Mills functional,

$$
\begin{aligned}
H= & \sum_{i=\text { odd }}-\frac{\omega\left(s^{3}\right)}{2} s_{i}^{2} d^{4}\left\langle P_{-} F_{A_{0}}\left(q_{i}\right), \phi_{f_{i}}^{*} g_{i} F_{-}(N) g_{i}^{-1}\right\rangle \\
& \left.+\sum_{j=\text { even }}-\frac{\omega\left(S^{3}\right)}{2} s_{j}^{2} d^{4} P_{+} F_{A_{0}}\left(q_{j}\right), \phi_{f_{j}}^{*} g_{j} F_{+}(N) g_{j}^{-1}\right\rangle \\
& +\sum_{j=\text { odd }} \sum_{\substack{j=\text { even } \\
j=[i \pm(2 l+1)] \bmod 2 k \\
0 \leq l \leq\left[1 / 2 d^{-2 / 5}\right]-1}}-Q \frac{\omega\left(s^{3}\right)}{2} s_{i}^{2} s_{j}^{2} d^{8} \cdot \operatorname{dist} .\left(q_{i}, q_{j}\right)^{-4} \\
& \cdot\left\langle\phi_{f_{i}}^{*} g_{i} F_{-}(N) g_{i}^{-1}, \phi_{f_{j}}^{*} g_{j} F_{-}(N) g_{j}^{-1}\right\rangle \\
& +\sum_{j=\mathrm{even}} \sum_{\substack{i=\text { odd } \\
i=[j \pm(2 l+1)] \bmod 2 k \\
0 \leq 2 l \leq\left[1 / 2 d^{-2 / s}\right]-1}}-Q \frac{\omega\left(s^{3}\right)}{2} s_{j}^{2} s_{i}^{2} d^{8} \cdot \operatorname{dist} .\left(q_{j}, q_{i}\right)^{-4} \\
& \cdot\left\langle\phi_{f_{j}}^{*} g_{j} F_{+}(N) g_{j}^{-1}, \phi_{f_{i}}^{*} g_{i} F_{+}(N) g_{i}^{-1}\right\rangle .
\end{aligned}
$$

Where $F_{ \pm}(N)$ are the curvatures at the north pole of the standard instanton or the standard anti-instanton over the round 4-sphere.

Suppose that at $\left\{\bar{g}_{i}\right\}_{1 \leq i \leq 2 k}$ the expressions below take the critical values (maximum)

$$
\begin{array}{ll}
-\left\langle P_{-} F_{A_{0}}, \phi_{f_{i}}^{*} \bar{g}_{i} F_{-}(N) \bar{g}_{i}^{-1}\right\rangle, & i=\text { odd } \\
-\left\langle P_{+} F_{A_{0}}, \phi_{f_{j}}^{*} \bar{g}_{j} F_{+}(N) \bar{g}_{j}^{-1}\right\rangle, & j=\text { even. }
\end{array}
$$

Fix $f_{i}=\bar{f}_{i}, 1 \leq i \leq 2 k$ such that $\phi_{\bar{f}_{i}}$ correspond to the coordinate system $\left\{s, y^{\alpha}\right\}_{\alpha=1}^{3}$ on the neighborhood $V_{0}$. According to the construction of the approximate solution $A(y)$, one can choose $\bar{g}_{i} \equiv \bar{g}_{j} \equiv \bar{g}$ with $\bar{g}$ independent of the positions of $\left\{q_{i}\right\}_{1 \leq i \leq 2 k}$ since $F_{A}$ is a harmonic 2-form valued in the Lie algebra of $S U(2)$. Set

$$
\left\{\begin{array}{l}
Q_{\text {odd }} \equiv Q_{i=\mathrm{odd}}=-\left\langle P_{-} F_{A_{0}}\left(\bar{q}_{i}\right), \phi_{f_{i}}^{*} \bar{g}_{i} F_{-}(N) \bar{g}_{i}^{-1}\right\rangle, \\
Q_{\text {even }} \equiv Q_{j=\mathrm{even}}=-\left\langle P_{+} F_{A_{0}}\left(\bar{q}_{j}\right), \phi_{f_{j}}^{*} \bar{g}_{j} F_{+}(N) \bar{g}_{j}^{-1}\right\rangle
\end{array}\right.
$$

Here $Q_{\text {odd }}$ and $Q_{\text {even }}$ are positive constants since by assumption $d\left\|F_{A_{0}}\right\|=0$ along the geodesic $C$ and $\left|P_{ \pm} F_{A_{0}}\right|>0$ on that geodesic, choose $\left\{q_{i}\right\}_{1 \leq i \leq 2 k}$ in the 
geodesic $C$ and dist. $\left(\bar{q}_{i}, \bar{q}_{i+1}\right)=\frac{\pi}{k}=d$. Set

$$
\bar{s}_{\text {odd }}^{2} \equiv \bar{s}_{i=\text { odd }}^{2}=Q_{j=\text { even }}\left\{Q\left\langle\bar{g} F_{+}(N) \bar{g}^{-1}, \bar{g} F_{+}(N) \bar{g}-1\right\rangle \sum_{l=0}^{\left[1 / 2 d^{-2 / 5}\right]-1} \frac{2}{(2 l+1)^{4}}\right\}^{-1}
$$

$$
=Q_{\text {even }}\left\{192 Q \sum_{l=0}^{\left[1 / 2 d^{-2 / 5}\right]-1} \frac{1}{(2 l+1)^{4}}\right\}^{-1},
$$

$$
\bar{s}_{\text {even }}^{2} \equiv \bar{s}_{j=\mathrm{even}}^{2}=Q_{\mathrm{odd}}\left\{192 Q \sum_{l=0}^{\left[1 / 2 d^{-2 / 5}\right]-1} \frac{1}{(2 l+1)^{4}}\right\}^{-1} .
$$

Here $\bar{s}_{\text {odd }}$ and $\bar{s}_{\text {even }}$ are constants which satisfy the following estimates:

$$
0<C_{1}<\bar{s}_{\text {odd }}, \bar{s}_{\text {even }}<C_{2} \text {, }
$$

where $C_{1}$ and $C_{2}$ are independent of $k$.

Set $\bar{y}=\left\{\left(\bar{s}_{i}, \bar{q}_{i}, \bar{g}_{i}\right)_{i=1}^{2 k}\right\} \in N_{2}$, here $\bar{s}_{i=\text { odd }}=\bar{s}_{\text {odd }}$ and $\bar{s}_{j=\text { even }}=\bar{s}_{\text {even. }}$. It is not hard to see that when $k$ is large enough $H$ at $\bar{y} \in N_{2}$ takes the critical values. The gradient of $H, \nabla H$, is close to the smooth section on $V \rightarrow N_{2}$. Actually, $\nabla H$ is first order term of the Taylor's expansion of the smooth section on $V \rightarrow N_{2}$. Set $f_{\xi}^{1}(y)=\nabla H, f_{\xi}^{2}(y)=f_{\xi}(y)-\nabla H$. In terms of the expansion of Yang-Mills functional restricted to the parameter space $N_{2}$, it is not difficult to check that $f_{\xi}^{2}$ is the higher order term. Suppose $H$ takes the critical value at $\bar{y} \in\left\{\left(\bar{s}_{i}, \bar{q}_{i}, \bar{g}_{i}\right)_{i=1}^{2 k}\right.$, then $f_{\xi}^{1}(\bar{y})=0$. By the direct calculation, $\left.\nabla f_{\xi}^{1}\right|_{\bar{y}}$ has the following expression:

$$
\begin{aligned}
\nabla f_{\xi}^{1}(\bar{y})= & Q \omega\left(s^{3}\right) \bar{s}_{\mathrm{odd}}^{2} \bar{s}_{\text {even }}^{2} d^{4}\left\{-192 C_{s} E_{2 k \times 2 k}\right. \\
& \oplus 128 C_{T^{1}}\left(2 \sum_{l=0}^{\left[1 / 2 d^{-2 / 5}\right]-1} \frac{1}{(2 l+1)^{4}} I_{2 k \times 2 k}-E_{2 k \times 2 k}\right) \\
& \oplus \sum_{\alpha=2}^{3} 128 C_{T^{\alpha}}\left(-2 \sum_{l=0}^{\left[1 / 2 d^{-2 / 5}\right]-1} \frac{1}{(2 l+1)^{4}} I_{2 k \times 2 k}-E_{2 k \times 2 k}\right) \\
& \oplus 960 C_{x^{1}}\left(-2 \sum_{l=0}^{\left[1 / 2 d^{-2 / 5}\right]-1} \frac{1}{(2 l+1)^{4}} I_{2 k \times 2 k}+E_{2 k \times 2 k}\right) \\
& \left.\oplus 960 C_{x^{t}}\left(2 \sum_{l=0}^{\left[1 / 2 d^{-2 / 5}\right]-1} \frac{1}{(2 l+1)^{4}} I_{2 k \times 2 k}-E_{2 k \times 2 k}\right)\right\} .
\end{aligned}
$$


Here $C_{s}, C_{T^{*}}$ and $C_{x^{t}}$ are positive constants, $I_{2 k \times 2 k}$ is identity, and $E_{2 k \times 2 k}$ is

$$
E_{i j}= \begin{cases}\frac{1}{|(i-j) \bmod 2 k|^{4}} & \text { if } i=\text { odd }, j=\text { even and } \\ 0 & |(i-j) \bmod 2 k| \leq 2\left[\frac{1}{2} d^{-2 / 5}\right]-1, \\ 0 & \text { for any other case. }\end{cases}
$$

By using the Fourier transformation, a priori estimates for the eigenvalues of $E_{2 k \times 2 k}$ are given as follows (cf. [27]):

Proposition 4.1. Let $E_{2 k \times 2 k}$ be the $2 k \times 2 k$ matrix which is defined by (4.10). For $E_{2 k \times 2 k}$, there exist the following properties:

1. The eigenvalues of $E_{2 k \times 2 k}$ can be written as follows:

$$
\begin{gathered}
\lambda_{i}=2 \sum_{l=0}^{\left[1 / 2 d^{-2 / 5}\right]-1} \frac{\cos \left[(i-1)(2 l+1) \frac{\pi}{k}\right]}{(2 l+1)^{4}}, \text { for } 1 \leq i \leq 2 k \\
\lambda_{\max }=2 \sum_{l=0}^{\left[1 / 2 d^{-2 / 5}\right]-1} \frac{1}{(2 l+1)^{4}}
\end{gathered}
$$

and

$$
\left|\lambda_{i}\right| \geq\left|\cos (i-1) \frac{\pi}{k}\right|, \quad \text { for } 1 \leq i \leq 2 k .
$$

2. If $k=$ even, it is not hard to see that $\lambda_{k / 2+1}=0$ and $\lambda_{3 k / 2+1}=0$ and the corresponding null space is spanned by the following two eigenvectors:

$$
\left\{\begin{array}{l}
u=(1,0,-1,0, \ldots, 1,0,-1,0) \\
v=(0,1,0,-1, \ldots, 0,1,0,-1)
\end{array}\right.
$$

3. If $k=$ odd, $E_{2 k \times 2 k}$ is a non-degenerate matrix.

The proof is referred to [27].

According to Proposition 4.1, a priori estimates for the Hessian $f_{\xi}^{1}=\nabla H$ are given as follows:

Proposition 4.2. Let $N_{2}(s, q, g)$ be a parameter space defined in Section 3. Fix $\bar{y}=\left\{\left(\bar{s}_{i}, \bar{q}_{i}, \bar{g}_{i}\right)_{i=0}^{2 k}\right\} \in N_{2}$ such that at which $H$ (the first order term of the Taylor's expansion of Yang-Mills functional) takes a critical value. Then the Hessian, $\nabla f_{\bar{\xi}}^{1}(\bar{y})=\left.\nabla^{2} H\right|_{\bar{y}}$, at $\bar{y}$ is of the following properties: 
1. When $k=$ even, $\nabla f_{\xi}^{1}(\bar{y})$ has a two dimensional null space which arises from the parameters $s_{i}$, and other non-zero eigenvalues are of the order $\mathcal{O}(d)$.

2. When $k=$ odd, the Hessian $\nabla f_{\xi}^{1}(\bar{y})$ contains five null eigenvectors which arise from the parameters $\left(q_{i}, T_{i}^{1}\right)$, and the order of other non-zero eigenvalues is $\mathcal{O}(d)$ too.

Proof. This is a direct consequence of Proposition 4.1, the proof is omitted.

We can use the gluing procedure developed in this section to establish a global setting. According to this global setting, we construct the approximate solution space $N_{2}(w, p, q, k)$ that involves many parameters, in our construction $N_{2}$ is diffeomorphic to

$$
\prod_{i=1}^{2 k}\left\{(0,1) \times F_{M}^{i} \times S U(2) S U(2)\right\} / \Gamma_{A_{0}} \times \Sigma_{k} \times \Sigma_{k}
$$

where $F_{M}^{i}$ are the frame bundles of bundles $\wedge_{ \pm}^{2} M$ of the self-dual and anti-selfdual 2-forms on $M, \Gamma_{A_{0}}$ is the isotropy group of the connection $A_{0}$, and $\Sigma_{k}$ is the symmetric group on $k$ letters. Then we can produce a new non-minimal solution as a small perturbation of some approximate solution $A$ in $N_{2}$.

THEOREM 4.3 (Theorem). Let $M$ be a compact oriented Riemannian 4manifold. Let $A_{0}$ be an isolated non-minimal Yang-Mills connection on $M$ with the structure group $S U(2)$ such that $d\left|P_{ \pm} F_{A_{0}}\right|=0$ along a simple closed geodesic and $\left|P_{ \pm} F_{A_{0}}\right|>0$ on this geodesic. Then there is a constant $K>0$ such that for any positive even $k>K$, there exists an irreducible non-minimal Yang-Mills $S U(2)$ connection with the same degree as $A_{0}$.

Remark here that the result above shows that, if we found an isolated nonminimal solution to the Yang-Mills equations on $M$, by using the gluing procedure and perturbation method we can construct an infinite number of gauge inequivalent irreducible $S U(2)$-connections over $M$ which are the nonminimal solutions with the same degree as the background connection. None of the solution found above are symmetric with respect to the Lie group actions on the base manifold $M$. In fact, our solutions have a uniform background curvature, with concentrations near points, spaced evenly along a geodesic. 
Near half of these points, the solution looks self-dual, and near the other half, it looks anti-self-dual.

One more remark that it is a technical condition that one requires that $d\left|P_{ \pm} F_{A_{0}}\right|=0$ and $\left|P_{ \pm} F_{A_{0}}\right|>0$ along a simple closed geodesic. Of course $S^{1} \times S^{3}$ and $S^{2} \times S^{2}$ are homogeneous spaces and the harmonic curvatures of background connections will satisfy that technical condition, so those examples in [27] are $O K$.

ProOF OF THEOREM 4.3. The strategy for proving the theorem above is to generalize the approach in [21]. Schematically, the approach is the following one. According to the preceding global setting, we have constructed a smooth section, $f_{\xi}$, of $V \rightarrow N_{2}$. Our method for finding the zero points of $f_{\xi}$ (i.e. Equation (4.4)) will be to decompose $f_{\xi}$ into $f_{\xi}^{1}+f_{\xi}^{2}$ and to reduce the question of finding the zero points of the given smooth section to the equations for the non-degenerate critical points for $H$.

Note that $f_{\xi}=f_{\xi}^{1}+f_{\xi}^{2}$ is a smooth section of $V \rightarrow N_{2}$. Suppose that $\bar{y}=\left\{\left(\bar{s}_{i}, \bar{q}_{i}, \bar{g}_{i}\right)_{i=1}^{2 k}\right\}$ is a critical point of $H$, then $f_{\xi}^{1}(\bar{y})=0$. According to Proposition 4.2 , when $k=$ even the tangent map $\nabla f_{\xi}^{1}(\bar{y})$ of $f_{\xi}^{1}$ contains two null eigenvectors

$$
\left\{\begin{array}{l}
u=(1,0,-1,0, \ldots, 1,0,-1,0), \\
v=(0,1,0,-1, \ldots, 0,1,0,-1) .
\end{array}\right.
$$

Let $V=\left\{t_{1} u+t_{2} v \mid\left(t_{1}, t_{2}\right) \in \mathscr{R}^{2}\right\}$ be the null space of $\nabla f_{\xi}^{1}(\bar{y})$ spanned by $u$ and $v$. Denote by $T_{\bar{y}}^{\perp} N_{2}$ the orthogonal complement of $T$ in $T_{\bar{y}} N_{2}$, it is non-degenerate and so can use the implicit function theorem such that $f_{\xi}=f_{\xi}^{1}+f_{\xi}^{2}$ is zero in the direction $T_{\bar{y}}^{\perp}$. Let $T=\left\{t_{1} u+t_{2} v|| t_{1}\left|+t_{2}\right|<\varepsilon\right\}$ be the small neighborhood of the origin at $V$, here $\varepsilon>0$ is small enough. For any $h(t)=\left\{t_{1} u+t_{2} v\right\} \in T$, let $\bar{y}(t)=\left\{\left(\bar{s}_{i}(t), \bar{q}_{i}, \bar{g}_{i}\right)_{i=1}^{2 k}\right\}$ such that $\left\{\bar{s}_{i}(t)\right\}_{1 \leq i \leq 2 k}=\left\{s_{i}\right\}_{1 \leq i \leq 2 k}+h(t)$. Hence $\bar{s}_{i}(0)=\bar{s}_{i}$. In terms of the implicit function theorem, by the small perturbation one may find $\tilde{y}^{\prime}(t)=\left\{\left(\tilde{s}_{i}^{\prime}(t), \tilde{q}_{i}^{\prime}(t), \tilde{g}_{i}^{\prime}(t)\right)_{i=1}^{2 k}\right\} \in N_{2}$ such that $f_{\xi}\left(\tilde{y}^{\prime}(t)\right)=0$ which is restricted to $T_{\bar{y}}^{\perp} N_{2}$. Therefore the problem of finding the zero points of $f_{\xi}(x)$ is reduced to the problem of finding the critical points of Yang-Mills functional restricted to the parameter space $T$.

If $|t|$ is small enough, then $\left|\bar{y}(t)-\tilde{y}^{\prime}(t)\right|$ is small too. Consider the Taylor's expansion of Yang-Mills functional restricted to $\tilde{y}^{\prime}(t)$ in the parameter $t$ : 


$$
\begin{aligned}
& Y M\left(\tilde{y}^{\prime}(t)=Y M\left(A_{0}\right)+16 \pi k^{2}\right. \\
& +\sum_{i=\text { odd }}-\frac{\omega\left(s^{3}\right)}{2} d^{4} Q_{\text {odd }} \bar{s}_{i}^{2}(t) \\
& +\sum_{\text {even }}-\frac{\omega\left(s^{3}\right)}{2} d^{4} Q_{\text {even }} \bar{s}_{j}^{2}(t) \\
& +\sum_{i=\text { odd }} \sum_{\substack{j=\text { even } \\
j=[i \pm 2(l+1)] \bmod 2 k \\
0 \leq l \leq\left[1 / 2 d^{-2 / 5}\right]-1}}-48 Q \omega\left(s^{3}\right) d^{4} \frac{\bar{s}_{i}^{2}(t) \bar{s}_{j}^{2}(t)}{|(i-j) \bmod 2 k|^{4}} \\
& +\sum_{j=\text { even }} \sum_{\substack{i=\text { odd } \\
i=2(j \pm 2(l+1)] \bmod 2 k \\
0 \leq l \leq\left[1 / 2 d^{-2 / 5}\right]-1}}-48 \omega\left(s^{3}\right) d^{4} \frac{\bar{s}_{j}^{2}(t) \bar{s}_{i}^{2}(t)}{|(j-i) \bmod 2 k|^{4}} \\
& +k C(t) d^{5 \frac{1}{5}} \ln d \\
& =Y M\left(A_{0}\right)+16 \pi^{2} k \\
& -\frac{\omega\left(s^{3}\right)}{2} k d^{4} Q_{\text {odd }}\left(\vec{s}_{\text {odd }}^{2}+t_{1}^{2}\right) \\
& -\frac{\omega\left(s^{3}\right)}{2} k d^{4} Q_{\text {even }}\left(\bar{s}_{\text {even }}^{2}+t_{2}^{2}\right) \\
& -96 k Q \omega\left(s^{3}\right) d^{4} \sum_{l=0}^{\left[1 / 2 d^{-2 / 5}\right]-1} \frac{\left(\bar{s}_{\text {odd }}^{2}+t_{1}^{2}\right)\left(\bar{s}_{\text {even }}^{2}+t_{2}^{2}\right)}{(2 l+1)^{4}} \\
& -96 k Q \omega\left(s^{3}\right) d^{4} \sum_{l=0}^{\left[1 / 2 d^{-2 / 5}\right]-1} \frac{\left(\bar{s}_{\text {even }}^{2}+t_{2}^{2}\right)\left(\bar{s}_{\text {odd }}^{2}+t_{1}^{2}\right)}{(2 l+1)^{4}} \\
& +k C(t) d^{5 \frac{1}{3}} \ln d .
\end{aligned}
$$

Since $\bar{s}_{\text {odd }}$ and $\bar{s}_{\text {even }}$ are chosen as follows

$$
\begin{aligned}
& \bar{s}_{\text {odd }}^{2}=Q_{\text {even }}\left\{192 Q \sum_{l=0}^{\left[1 / 2 d^{-2 / 5}\right]-1} \frac{1}{(2 l+1)^{4}}\right\}^{-1} \\
& \bar{s}_{\text {even }}^{2}=Q_{\text {odd }}\left\{192 Q \sum_{l=0}^{\left[1 / 2 d^{-2 / 5}\right]-1} \frac{1}{(2 l+1)^{4}}\right\}^{-1} .
\end{aligned}
$$


Hence, one as

$$
\begin{aligned}
Y M\left(\tilde{y}^{\prime}(t)\right)= & Y M\left(A_{0}\right)+16 \pi^{2} k \\
& -96 \omega\left(s^{3}\right) k Q d^{4}\left(\bar{s}_{\text {odd }}^{2}+t_{1}^{2}\right) t_{2}^{2} \sum_{l=0}^{\left[1 / 2 d^{-2 / 5}\right]-1} \frac{1}{(2 l+1)^{4}} \\
& -96 \omega\left(s^{3}\right) k Q d^{4}\left(\bar{s}_{\text {even }}^{2}+t_{2}^{2}\right) t_{2}^{1} \sum_{l=0}^{\left[1 / 2 d^{-2 / 5}\right]-1} \frac{1}{(2 l+1)^{4}} \\
& +k C(t) d^{5 \frac{1}{5}} \ln d \\
= & Y M\left(F_{A_{0}}\right)+16 \pi^{2} k \\
& -96 \omega\left(s^{3}\right) Q d^{4} k \sum_{l=0}^{\left[1 / 2 d^{-2 / 5}\right]-1} \frac{1}{(2 l+1)^{4}}\left\{\bar{s}_{\text {even }}^{2} t_{1}^{2}+\bar{s}_{\text {odd }}^{2} t_{2}^{2}+2 t_{1}^{2} t_{2}^{2}\right\} \\
& +k C(t) d^{5 \frac{1}{5}} \ln d . \quad
\end{aligned}
$$

In terms of the expansion given above, by using the implicit function theorem one may find small $\bar{t}=\left(\bar{t}_{1}, \bar{t}_{2}\right) \in T$ such that $\tilde{y}=\tilde{y}^{\prime}(\bar{t})=\left\{\left(\tilde{s}_{i}^{\prime}(\bar{t}), \tilde{q}_{i}^{\prime}(\bar{t}), \tilde{g}_{i}^{\prime}(\bar{t})\right)_{i=1}^{2 k}\right\} \in N_{2}$ is a critical point of Yang-Mills functional, i.e. $f_{\xi}(\tilde{y})=0$. Therefore, for sufficiently large even integer $k$, one may find a non-minimal solution, $A(\tilde{y})$, to the Yang-Mills equations on $M$ with the same degree as the background connection $A_{0}$. The proof is complete.

Remark that as a direct consequence of the above theorem, one can construct an infinite number of non-minimal irreducible connections over $S^{2} \times S^{2}$ and $S^{1} \times S^{3}$, for details, refer to [27]. For the base manifold $S^{1} \times S^{3}$, the existence of non-minimal solutions to the Yang-Mills equations has already been established by T. Parker [12] via different methods. His basic technique is to consider with a symmetric group action that reduces the dimensionality of Yang-Mills equations, and to use the min-max argument.

\section{Some Remarks}

We have established a method for finding the non-minimal solutions to the Yang-Mills equations on the generic 4-manifolds. In our construction, the key step is to find an isolated non-minimal Yang-Mills connection as a background connection. In fact, for a generic 4-manifold $M$, it is possible that there are no reducible self-dual or the anti-self-dual connections over $M$. D. Freed and $\mathrm{K}$. Uhlenbeck pointed out in [10] that if the intersection matrix of a 4-manifold is indefinite, then for an open dense set of metrics on the manifold, there are no 
line bundle solutions to the self-dual or anti-self-dual equations. Of course, there are still solutions to the Yang-Mills equations in this case by the Hodge theory, so the indefinite case provides an example where the topological lower bound of Yang-Mills functional is not attained and there exist the non-minimal, reducible $S U(2)$ Yang-Mills connections which are isolated or become a compact manifold.

But, in general, the condition that $d\left|P_{ \pm} F_{A}\right|=0$ along a closed geodesic can not be satisfied. For the general cases, we have only partial answer. If one knew that $\left|F_{A}\right|$ never vanished on $M$, then one could conformally change the metric to make $\left|F_{A}\right|$ constant, and find the Yang-Mills fields for this new metric; since the Yang-Mills equations are conformally invariant these would be the Yang-Mills fields for the original metric.

There is an obstruction to having $\left|F_{A}\right|$ never vanish on $M$. A theorem of Hirzebruch and Hopf (Math. Ann. 136 (1958), 156-172) shows that there is no non-vanishing section of $\wedge^{2} T^{*} M$ unless $3 \tau+2 \chi$ and $3 \tau-2 \chi$ lie in $\Omega=\left\{I(W, W) \mid W \in H^{2}(M), W=\tilde{W}_{2}+K, K \in 2 H^{2}(M)\right\}$ where $\tau$ and $\chi$ are the signature and Euler class of $M, \tilde{W}_{2}$ is an integral lift of the second StiefelWhitney class, and $I($,$) is the intersection form on the 4-manifold M$. If this condition is not true then $F_{A} \in \Gamma\left(\wedge^{2} T^{*} M\right)$ must vanish somewhere.

It is a long-standing question whether there exist the Yang-Mills fields over $S^{4}$ which are neither self-dual nor anti-self-dual. Until recently, the only known the finite-energy solutions to the Yang-Mills equations on the round 4-sphere were the self-dual and anti-self-dual solutions [1], [2]. Now, L. \& R. Sibner and K. Uhlenbeck [20]; T. Parker [12]; L. Sadun and J. Segert [16], [17] and G. Bor [6] have found the non-minimal solutions over $S^{4}$ via the reduction of dimensionality. In particular, Sadun and Segert [16], [17] considered the quadrapole bundles which are a family of $S U(2)$-equivariant quaternionic line bundles over $S^{4}$ that originally arose in quantum mechanics. They used the explicit O.D.E. computations to show the most of these bundles admit no selfdual or anti-self-dual Yang-Mills fields (Similarly, one can get same results by the equivariant Morse theory [13].). More precisely, they proved the following results:

For any $C_{2} \neq \pm 1$, there exists at least one non-minimal solution to the Yang-Mills equations on the round 4-sphere $S^{4}$.

It is worth remarking here that we do not know whether any exists for $C_{2}= \pm 1$.

We guess that our theorem can be applied to the construction of Sadun and Segert. If one choose Sadun and Segert's equivariant connections as the 
background connections, then the better existences may hold. It is stated below:

For any $C_{2} \neq \pm 1$, there exists in infinite number of non-minimal $S U(2)$ Yang-Mills connections over the round 4-sphere $S^{4}$.

We would like to point out that in order to use our construction, one requires the following technical lemma.

LeMma 5.1. The moduli space of non-minimal SU(2)-Yang-Mills connections over $S^{4}$ given by Sadun and Segert should become the isolated manifolds which depend only on the conformal diffeomorphic transformation group of $S^{4}$.

There are a number of possible approaches for proving the lemma given above. We have not yet been able to push any of these through to fruition. We shall review one fairly convincing approach briefly.

The most convincing approach so far is the direct method of the calculus of variations. What one has to do is studying the null spaces of the Hessian of Yang-Mills functional at these background connections by the separation of variables (cf. [26], [27]).

First, we briefly recall the main features of Sadun and Segert's construction [17]. Sadun and Segert consider the connections that are equivariant with respect to a symmetric group $S U(2)$, that acts on $S^{4} \subset \mathscr{R}^{5}$ via the unique irreducible representation. The principal orbits are three-dimensional, reducing the Yang-Mills equations and the self-dual equations to the systems of ordinary differential equations on an interval with certain singularities at the endpoints. They first prove the existence theorem for the systems reduced from the YangMills equations. Then they are able to exclude the self-dual solutions out of list of equivariant solutions.

Now let us return to Lemma 5.1. According to Sadun and Segert's construction, the principal orbits are three-dimensional, and there are two exceptional orbits of dimension two; meanwhile there exists a closed geodesic segment of length $\pi / 3$ that intersects each $S U(2)$-orbit exactly once [17]. Hence, Sadun and Segert's non-minimal Yang-Mills connections may be viewed as the $S U(2)-$ equivariant solutions of Dirichlet boundary value problems for the Yang-Mills connections over a compact 4-manifold with the smooth boundaries, the equations in the interior are the full Yang-Mills equations [11]. We should study the eigenvalues of Dirichlet problems on the compact 4-manifold with the smooth boundaries. In particular, we are interested in the null spaces. Our basic method is still to use the direct calculation by the separation of variables [26]. Usually, it is more complicated. 
On the other hand, we note that the Yang-Mills equations are invariant under the action of the conformal diffeomorphic transformation group of the base manifold. Thus the orbit of non-minimal Yang-Mills connection under the action of the conformal diffeomorphic transformation group of the base manifold is a family of non-minimal Yang-Mills connections. Since Sadun and Segert's connections are the $S U(2)$-equivariant connections over $S^{4}$. The symmetric group $S U(2)$ is an isotropy subgroup of the conformal diffeomorphic transformation group of $S^{4}$. Hence Sadun and Segert's connections are of at least twelve-dimensional parameters [18].

Finally, we make some remarks before ending this article. In order to use our construction, the key ingredient is that the background connections are isolated points or isolated finite dimensional smooth manifolds. In principle a good understanding of the parameter spaces for the non-minimal solutions to the Yang-Mills equations would enable one to use the Morse theory for finding the higher critical points of Yang-Mills functional, or at least to get qualitative information about them.

Question. Are the moduli spaces of non-minimal solutions of Yang-Mills equations isolated points or isolated finite dimensional manifolds which depend only on the conformal diffeomophic transformation group of the base manifold?

The progress in understanding these spaces has been made recently be Freed and Uhlenbeck [10], Wang [26], [27], and Sadun and Segert [18]. It seems very likely that the answer to Question is affirmative, at least for cases $S^{2} \times S^{2}$ and $S^{1} \times S^{3}$ [26], [27]. Also, Sadun [15] used the numerical method to discover that, after the gauge fixing, each component of the non-self-dual moduli spaces constructed by Sadun and Segert appears to consist only of the conformal copies of a single solution.

\section{References}

[1] Atiyah, M. F., Hitchin, N., and Singer, I. M.: Self-duality in four-dimensional Riemannian geometry. Proc. Roy. London Ser. A 362 (1978), 452-461.

[2] Atiyah, M. F., Jones, J. D. S.: Topological aspects of Yang-Mills theory. Comm. Math. Phys. 61 (1978), 97-118.

[3] Bahri, A.: Critical points at infinity in some variational problems. Pitman Research Notes in Math. Ser. 182, Longman Sci. and Tech., 1988.

[ 4 ] Bahri, A., Berestyski, H.: Forced vibrations in super quadric Hamiltonian systems. Acta Math. 152 (1984), 143-197.

[ 5] Bahri, A., Coron, J. M.: On a nonlinear elliptic equation involving the critical Sobolev exponent: the effect of the topology of the domain. Comm. Pure Appl. Math. 41 (1988), 253-294. 
[6] Bor, G.: Yang-Mills fields which are not self-dual. Comm. Math. Phys. 145 (1992), 393-410.

[7] Bourguignon, J. P., Lawson, H. B., Jr.: Stability and isolation phenomena for Yang-Mills equations. Comm. Math. Phys. 79 (1981), 189-230.

[ 8 ] Donaldson, S.: Connection, cohomology, and the intersection forms of 4-manifolds. J. Diff. Geom. 24 (1986), 275-341.

[9] Donaldson, S., Kronheimer, P.: The geometry of four-manifolds. Oxford Univ. Press, 1990.

[10] Freed, D., Uhlenbeck, K.: Instantons and four-dimensional manifolds. Springer, Berlin, 1984.

[11] Marini, A.: Dirichlet and Neumann boundary value problems for Yang-Mills connections. Comm. Pure Appl. Math. 45 (1992), 1015-1050.

[12] Parker, T.: Non-minimal Yang-Mills fields and dynamics. Invent. Math. 107 (1992), 397-420.

[13] Parker, T.: A Morse theory for equivariant Yang-Mills. Duke Math. J. 66 (1992), 337-356.

[14] Seldlacek, S.: A direct method for minimizing the Yang-Mills functional over 4-manifolds. Comm. Math. Phys. 86 (1982), 515-527.

[15] Sadun, L.: A symmetric family of Yang-Mills fields, preprint.

[16] Sadun, L., Segert, J.: Non-self-dual Yang-Mills connections with nonzero Chern number. Bull. Amer. Math. Soc. 24 (1991), 163-170.

[17] Sadun, L., Segert, J.: Non-self-dual Yang-Mills connections with quadrapole symmetry. Comm. Math. Phys. 145 (1992), 363-391.

[18] Sadun, L., Segert, J.: Stationary points of the Yang-Mills action. Comm. Pure Appl. Math. 145 (1992), 461-484.

[19] Sacks, J., Uhlenbeck, K.: On the existence of minimal immersions of 2-spheres. Ann. Math. 113 (1981), 1-24.

[20] Sibner, L., Sibner, R. and Uhlenbeck, K.: Solutions to Yang-Mills equations which are not selfdual. Proc. Natl. Acad. Sci. USA 86 (1989), 8610-8613.

[21] Taubes, C. H.: Morse theory and monopoles: topology in long range forces. Progress in gauge field theory. ed. G. 't Hooft et al. NATO ASI Ser. Series B, Phys. Vol. 115 (1983).

[22] Taubes, C. H.: Self-dual connections on 4-manifolds with indefinite intersection matrix. J. Diff. Geom. 19 (1984), 517-560.

[23] Taubes, C. H.: Path-connected Yang-Mills spaces. J. Diff. Geom. 19 (1984), 337-392.

[24] Taubes, C. H.: A framework for Morse theory for the Yang-Mills functional. Invent. Math. 94 (1988), 347-402.

[25] Uhlenbeck, K.: Connections with $L^{p}$-bounds on curvature. Comm. Math. Phys. 83 (1982), 3142.

[26] Wang, H.: The construction of isolated reducible $S U(2)$-connections over $S^{2} \times S^{2}$. Acta Math. Sinica, New Series, 8 (1992), 60-77.

[27] Wang, H.: The existence of non-minimal solutions to the Yang-Mills equation with group $S U(2)$ on $S^{2} \times S^{2}$ and $S^{1} \times S^{3}$. J. Diff. Geom. 34 (1991), 701-767.

[28] Wente, H.: Counterexample to a conjecture of H. Hopf. Pacific. J. Math. 121 (1986), 193-243.

\author{
Hong-Yu Wang \\ Department of Mathematics \\ National University of Singapore \\ Singapore 119260
}

